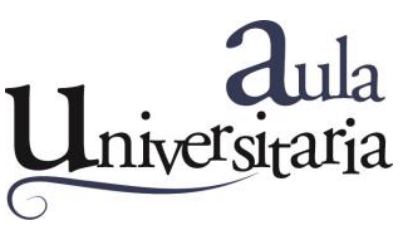

\title{
Los desafíos de la incorporación de TIC en la educación universitaria
}

\author{
FABRO, Ana Patricia ${ }^{1}$
}

Filiaciones institucionales

${ }^{1}$ Cátedra de Morfología Normal. Facultad de Bioquímica y Ciencias Biológicas. Universidad Nacional del Litoral.

\section{Correspondencia}

Paraje El Pozo, s/n, 1er. Piso. Santa Fe de la Vera Cruz, Santa Fe, Argentina. Tel. 54-342-4575210

anapfabro@hotmail.com

\section{Resumen}

Inmersas en la «sociedad del conocimiento», las instituciones educativas transitan el desafío de incorporar las TIC en el marco de enfoques pedagógicos activos que posibiliten «aprender a aprender», razón por la que el rumbo de la educación se transforma de un sistema clásico y conservador a un ambiente dinámico y creativo. La presencia y facilidad para el uso de medios interactivos en la educación permiten que los estudiantes adquieran competencias para transformar la información en conocimiento. La actual tendencia educativa está encaminada hacia la elaboración de sistemas interactivos que posibiliten a los alumnos concentrarse en el razonamiento y en la resolución de problemas, dejando atrás modelos tradicionales de enseñanza centrados en la transmisión de conocimientos.

\section{Palabras clave}

desafíos | TIC | educación

\section{Summary}

The educational institutions face at present the challenge of ICT incorporation to their learning activities. This occurs in the framework of the "society of knowledge" where education, instead of being classic and conservative as usual, requires active pedagogical approaches enabling «learning to learn» as well as dynamic and creative environments. In this context, interactive media in education facilitate the transformation 
of information into knowledge through the acquisition of the corresponding competences. Current educational trend is based on the elaboration of interactive systems preparing students for reasoning and solving problems and leaving behind traditional models of teaching particularly centred on passive knowledge reception.

\section{Keywords}

challenges / ICT / education

En la actualidad, las TIC dinamizan cambios que involucran al conjunto de la vida social. Sin embargo, más allá de los promisorios discursos que interpelan a hombres y mujeres del globo como "consumidores y usuarios de un mundo tecnologizado", la integración de las TIC en los distintos países, regiones geográficas y grupos sociales no se da de manera uniforme (Batista y col., 2007).

Como centros generadores de conocimientos, las universidades están llamadas a cumplir un rol fundamental en este proceso de cambio, actualizando y asimilando estas tecnologías para alcanzar un alto nivel de calidad y pertinencia, ya que las mismas facilitan el acceso a la información científica y técnica a partir de recursos informáticos y de telecomunicaciones, insertándose en el área educativa y de investigación (Vargas, 2005).

Asimismo, el desarrollo sin precedentes de las tecnologías digitales de la información y de la comunicación, está llevando a centrar una buena parte del interés de los educadores en el potencial de sus aplicaciones, para dar respuesta a los desafíos que hoy tiene planteados la educación (De Pablos Pons, 2009).

Al respecto, Marqués Graells (2000) afirma que se hace necesario conocer si la aplicación de estas tecnologías en el ámbito educativo genera experiencias de aprendizaje, promueve en los estudiantes procesos de comprensión y construcción de conocimientos, y modos de pensar inteligentes, creativos y profundos, que favorezcan su desarrollo personal y profesional.

En las últimas décadas, las TIC se han convertido en parte de nuestra vida diaria, han transformado de una u otra forma la manera de comunicarnos y de procesar la información en nuestra sociedad. Se encuentran en todos los ámbitos, ya sean públicos o privados, y transforman las relaciones del ser humano. La educación no está ajena a esta transformación ya que las TIC están ofreciendo nuevas alternativas para la enseñanza y los aprendizajes. Actualmente, la difusión de las mismas parece abrir nuevas perspectivas para la ampliación del espacio público del conocimiento. Sin embargo, a pesar de la amplia difusión de las TIC, aún persiste una preocupante brecha digital que se da entre los dos hemisferios, entre los distintos países, así como entre diferentes comunidades de un mismo país.

Las disparidades en el acceso y apropiación de las TIC son crecientes en todos los órdenes y dificultan el desarrollo de numerosos países y comunidades, ejemplo de ello es que la mitad de la población mundial nunca ha realizado una 
llamada telefónica, y en Tokio hay más teléfonos que en todo el continente africano. Solo el $11 \%$ de la población mundial tiene acceso a internet y el $90 \%$ de ellos vive en países industrializados (UNESCO, 2009). En Argentina, por ejemplo, casi las dos terceras partes de las conexiones a Internet se realizan en la Ciudad Autónoma de Buenos Aires y en la provincia de Buenos Aires (Azinián, 2009).

Para contribuir a disminuir esta brecha digital y poder concretar en los hechos la evolución de la «sociedad de la información» a la «sociedad del conocimiento», es preciso desarrollar una alfabetización múltiple, de carácter crítico-reflexivo que trascienda lo puramente instrumental y tenga como objetivo prioritario la capacitación, para convertir la información en conocimiento, y hacer del conocimiento un elemento de colaboración para la transformación de la sociedad (Gutiérrez Martín, 2003).

En el mismo sentido, Edgar Morin (2009) expresa que en la era de la "sociedad del conocimiento» se hace necesario la puesta en práctica de lo que él denomina el diezmo epistemológico: es decir destinar el diez por ciento de los presupuestos en educación para financiar la reflexión sobre el valor y la pertinencia de lo que se enseña. Es decir, no se puede utilizar a las TIC como meros instrumentos para la búsqueda y procesamiento de la información, sino que se debe poner énfasis en reflexionar sobre esa información a los fines de construir conocimiento.

En la actualidad existe un creciente interés por usar TIC como recurso pedagógico, hay una motivación y un optimismo importante, pero también ello implica un desafío. Las TIC no fueron creadas para fines educativos, sino con el fin de comunicar información. ¿Qué implica esto? Implica el reto de crear usos educativos inteligentes a tecnologías que no fueron proyectadas con fines pedagógicos. Ello implica una tarea no trivial, una tarea complejísima para quienes pensamos que la tecnología en educación tiene su razón de ser en tanto constituya un valor educativo agregado que justifique su uso, en tanto aporte algo distinto, nuevo y de valor, en relación con estrategias utilizadas anteriormente. En consecuencia para que la inserción de las TIC en educación no quede subyugada al campo de las utopías, sino que pueda desarrollar todas sus potencialidades, es necesario conocer cuál es el contexto educativo en donde se aplican y de qué manera participan los destinatarios en las innovaciones realizadas.

Inmersas en la «sociedad del conocimiento» las instituciones educativas transitan el desafío de incorporar las TIC en el marco de enfoques pedagógicos que posibiliten «aprender a aprender», razón por la que el rumbo de la educación se transforma de un sistema clásico y conservador a un ambiente dinámico y creativo. La presencia y facilidad para el uso de medios interactivos en la educación, permiten que el alumno desarrolle competencias para convertir la información en conocimiento. La actual tendencia educativa está encaminada hacia la elaboración de sistemas interactivos que permitan a los alumnos concentrarse en el razonamiento y en la resolución de problemas. 
Así mismo es preciso reflexionar acerca de las palabras de Manuel Area Moreira (2003) quien señala que el proceso de aprendizaje universitario no puede consistir en la mera recepción y memorización de datos recibidos en la clase, sino en la permanente búsqueda, análisis y reelaboración de informaciones obtenidas en las redes.

Por otra parte, si bien los alumnos ingresantes a la Educación Superior en Argentina pertenecen a la generación de los llamados «nativos digitales», ${ }^{1}$ estos jóvenes no siempre utilizan tecnologías de la información y la comunicación para sus aprendizajes (Fabro, 2015).

Sobre este tema, Diego Levis (2011) sostiene que en nuestro país el estudiante se siente a gusto recibiendo un método de enseñanza tradicional, y en ocasiones no está dispuesto a participar del uso de tecnología si no lo considera necesario, conducta que viene arraigada desde su educación básica.

El mismo autor expresa que en Argentina, el sistema educativo formal, desde sus niveles más básicos, lleva al estudiante a estar arraigado a un modelo de enseñanza que no es funcional en su totalidad, ya que le facilita todo al alumno y no lo hace ser partícipe de su propia educación, lo que lo conduce a un estado de confort, en el cual su interés es el de recibir información, sin preocuparse por su obtención.

Si se suma a esto la tendencia del profesorado de enseñar desde una metodología tradicional y la falta de preparación de los profesores para integrar nuevos recursos a la enseñanza, se genera un contrasentido en la educación actual debido a que se dispone de numerosas posibilidades para el aprendizaje mediado por tecnologías, pero paradójicamente los estudiantes siguen prefiriendo la enseñanza tradicional.

En consecuencia, el impacto educativo de las TIC no se produce solo por la incorporación de las mismas al aula, sino que tiene lugar cuando los sujetos implicados se esfuerzan en conocer e interpretar las nuevas posibilidades educativas que ofrecen dichas tecnologías y cuando intentan crear y desarrollar nuevas estrategias de enseñanza, a los fines de potenciar el aprendizaje activo, participativo y reflexivo de los estudiantes mediado por tecnologías.

\footnotetext{
1 Término propuesto por Marc Prensky (2001) en su libro Digital natives, digital inmigrants, para definir a los jóvenes que desde temprana edad utilizan tecnologías de la información en su vida diaria.
} 


\section{Referencias bibliográficas}

- Area, Moreira, M. (2009). Introducción a la tecnología educativa. Universidad de La Laguna (pp 1-78). https://campusvirtual.ull.es/ocw/file.php/4/ebookte.pdf

- Azinián, H. (2009). Las tecnologías de la información y la comunicación en las prácticas pedagógicas. Noveduc.

- Batista, M.A.; Celso, V.E. y Usubiaga, G.G. (2007). Tecnologías de la información y la comunicación en la escuela: trazos, claves y oportunidades para su integración pedagógica. Ministerio de Educación, Ciencia y Tecnología de la Nación.

- De Pablos Pons, J. (2009). Tecnología educativa. 1era. ed. Aljibe.

- Fabro, A. (2015). Contribución de las tecnologías de la información y la comunicación (TIC) a la enseñanza y los aprendizajes de las Ciencias Morfológicas. Tesis Doctoral.

- Gutiérrez, M. (2003). Alfabetización digital: Algo más que ratones y teclas. Gedisa.

- Levis, D. (2011). Redes educativas. Medios sociales, entornos colaborativos y procesos de enseñanza y aprendizaje. Universities and Knowledge Society Journal, 8(1), 7. Universitat Oberta de Catalunya Barcelona. http://www.redalyc.org/pdf/780/78017126002.pdf

- Marqués Graells, P. (2000). Nueva cultura, nuevas competencias para los ciudadanos. La alfabetización digital. Roles de los estudiantes hoy. Universidad Autónoma de Barcelona.

- Morin, E. (2009). Los siete saberes necesarios para la educación del futuro. Paidós lberoamérica.

- Prensky, M. (2001). Digital natives, digital inmigrants. MCB University Press, 9(5), 1-6. http://www.marcprensky.com/writing/Prensky\%20-\%20Digital\%20Natives,\%20Digital\%20Immigrants\%20-\%20Part1.pdf

- UNESCO (2009). Hacia las sociedades del conocimiento. http://www.uned.es/ntedu/espanol/novedades/Sociedades_conocimiento.pdf

- Vargas, M. (2005). Las Tecnologías de la Información y la Comunicación (TIC) herramientas viabilizadoras para el Acceso y Difusión de Información Científica. Orbis: revista de Ciencias Humanas, 1(1), 35-51. 